\title{
Applying Gravitational Lensing to Neutrino Flux
}

\author{
Lichuan $\mathrm{Xu}^{*}$ \\ Shenzhen Yucai High School, Shenzhen 518000, Guangdong, China \\ *Corresponding Author email: Glutate@outlook.com
}

Keywords: neutrino flux; gravitational lensing; neutrino oscillation; Python

\begin{abstract}
In this paper, neutrinos' behavior when interacting with gravity is discussed. Specifically, effect of applying gravitational lensing, which is often applied to visible light, to neutrino flux is discussed. Formalism of applying gravitational lensing to photons is used to approximate neutrinos' behavior, followed by a study of magnification of neutrino flux and a study of neutrino oscillation in this case.
\end{abstract}

\section{Introduction}

Interacting with neutrinos via weak interaction, like absorbing neutrinos significantly using ordinary material, which has very small cross-section, demands extremely large quantity of absorber [1]. For this reason, neutrino telescopes are often built as large containers of absorbing material (e.g. Super-Kamiokande, which holds 50000 tons of ultrapure water)[2]. Even though astrophysical neutrino sources like supernovae generate large neutrino fluxes, only a tiny fraction of the neutrinos can be detected by neutrino telescopes [3]. This means that more distant sources would be very hard to detect. One way of observing more distant sources would be using one of the effects of gravity [4]: gravitational lensing. This effect allows massive objects like galaxies or galaxy clusters to gravitationally deflect the paths of neutrinos emitted by distant sources [5]. When multiple path lines can be deflected in the direction of the observer, the flux of neutrinos would be magnified, helping observers on Earth to detect much more distant sources.

\section{Introduction to Thin Lens Approximation}

\subsection{Deflection angle}

Section Headings. To simplify the analysis, some assumptions are made in order to get some useful conclusions. Assuming the overall geometry of the universe to be well described by the Robertson-Walker metric, Newtonian potential to be small, and the lens's peculiar velocity to be much smaller than the speed of light, would significantly simplify the deduction. Since the area where the deflection takes place is small compared with the distance between the light source, the lens, and the observer, it can be assumed that the light keeps unperturbed before and after the deflection. This will also be the prerequisite of the discussion of neutrino oscillation in section 4.2. With the assumptions mentioned above, the deflection of a light ray would be given by

$$
\overrightarrow{\hat{\alpha}}=\frac{2}{c^{2}} \int \vec{\nabla}_{\perp} \Phi d l
$$

where $\Phi$ is Newtonian potential and the integral is taken along photon trajectory.

With more assumptions and treating the lens as a point mass, we can get the deflection angle, by which the ray is deflected,

$$
\hat{\alpha}=\frac{4 G M}{c^{2} b}
$$

where $b$ is the impact parameter of the unperturbed ray, $M$ is the lens mass.

Due to the discrepancy between the distance within which the deflection occurs and the total 
length of the light path, the lens can be simplified as a mass sheet, whose plane is called the lens plane. Considering a circularly symmetry lens, we have

$$
\hat{\alpha}(\xi)=\frac{4 G M(\xi)}{c^{2} \xi}
$$

where $\xi$ is the distance from the lens center and $M(\xi)$ is the mass enclosed within radius $\xi$.

Introducing the reduced deflection angle

$$
\vec{\alpha}=\frac{D_{d s}}{D_{s}} \overrightarrow{\hat{\alpha}}
$$

where $D_{d s}$ and $D_{s}$ are the angular-diameter distances between lens and source, observer and source, respectively. we will have the lens equation

$$
\vec{\beta}=\vec{\theta}-\vec{\alpha}(\vec{\theta})
$$

where $\vec{\beta}$ and $\vec{\theta}$ are respectively the angular position of the source and the image.

Setting $\xi=D_{d} \theta$ and considering a lens with a constant surface-mass density, the reduced deflection angle will be

$$
\alpha(\theta)=\frac{4 \pi G \Sigma}{c^{2}} \frac{D_{d} D_{d s}}{D_{s}} \theta
$$

where $D_{d}$ is the angular-diameter distance between the observer and the lens.

\subsection{Critical surface-mass density}

If a lens focuses perfectly with a well-defined focal length, which means that all the deflected neutrinos would be brought to a point. To study this condition, $\beta$ should be 0 , i.e. $\alpha(\theta)=\theta$. The solution of equation

$$
\frac{4 \pi G \Sigma}{c^{2}} \frac{D_{d} D_{d s}}{D_{s}}=1
$$

is

$$
\Sigma_{c r}=\frac{c^{2}}{4 \pi G} \frac{D_{s}}{D_{d} D_{d s}}
$$

where $\Sigma_{c r}$ is defined as a critical surface-mass density.

\subsection{Magnification factor}

Considering a circularly symmetric lens with an arbitrary mass profile, we have:

$$
\beta(\theta)=\theta-\frac{D_{d s}}{D_{d} D_{s}} \frac{4 G M(\theta)}{c^{2} \theta}
$$

If a source lies exactly on the optical axis $(\beta=0)$, it will be imaged as a ring if the lens is supercritical $\left(\Sigma>\Sigma_{c r}\right)$. Assuming the lens to be a point mass, the solution of the ring's radius, namely the Einstein radius, will be:

$$
\theta_{E}=\sqrt{\frac{4 G M}{c^{2}} \frac{D_{d s}}{D_{d} D_{s}}}
$$

And the magnification factor

$$
\mu=\frac{u^{2}+2}{u \sqrt{u^{2}+4}}, u=\frac{\beta}{\theta_{E}}
$$




\section{Magnification of neutrino flux}

One of the most useful properties of gravitational lens is that they can converge light to facilitate observation of far and faint celestial bodies. Similarly, gravitational lensing can also amplify neutrino flux, therefore benefiting neutrino observation.

To visualize and study the effect of magnification, Python is used to plot some figures, showing the extent to which neutrino flux is magnified.

A neutrino gravitational lensing model is built for study. A Cartesian coordinate system is built on a plane to be a frame of reference. A neutrino source is located at $(-50000 \mathrm{Mpc}, 0)$; a point mass which has a typical galactic mass is located at the origin, acting as a gravitational lens; the observer is located at an arbitrary point on the first quadrant. The source, the point mass, and the observer in this model are all motionless with respect to the plane. For each location of the observer, the $\mathrm{x}$ and $\mathrm{y}$ coordinates are input as the independent variables, and some physical quantities at this location are calculated according to the formulas in section 2.3. and showed as the dependent variable $\mathrm{z}$.

Critical parameters of this model are shown in Appendix 1, and the source code of these figures is showed in Appendix 2.
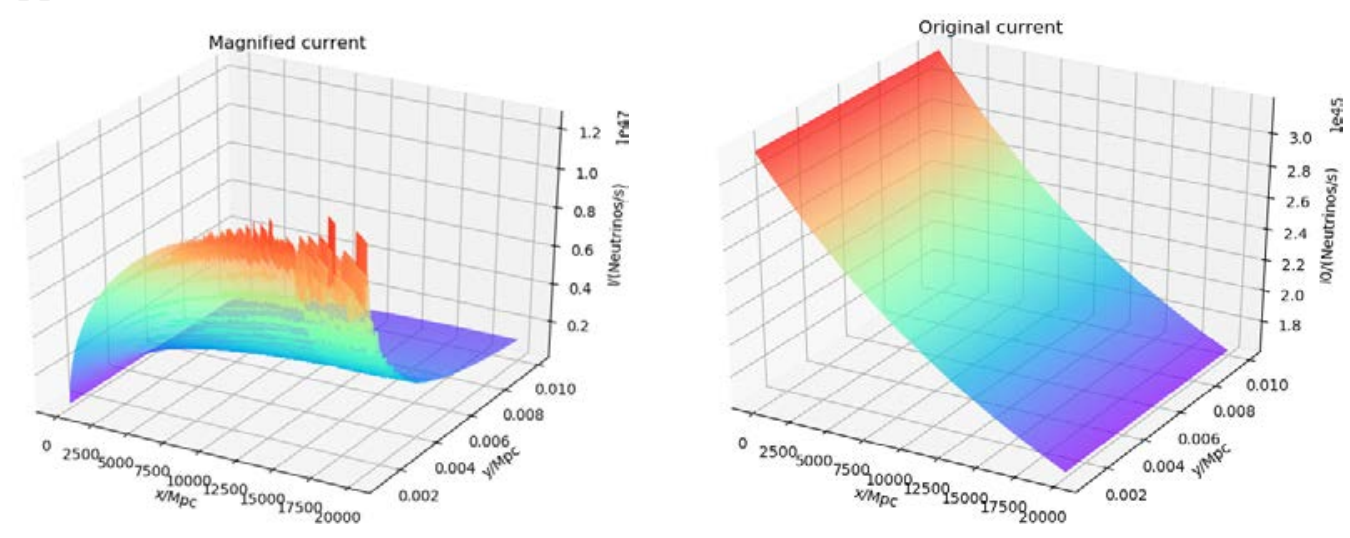

Fig.1

The left figure shows the original current of neutrino flux without gravitational lensing, while the right one shows the current of converged and therefore magnified neutrino flux.

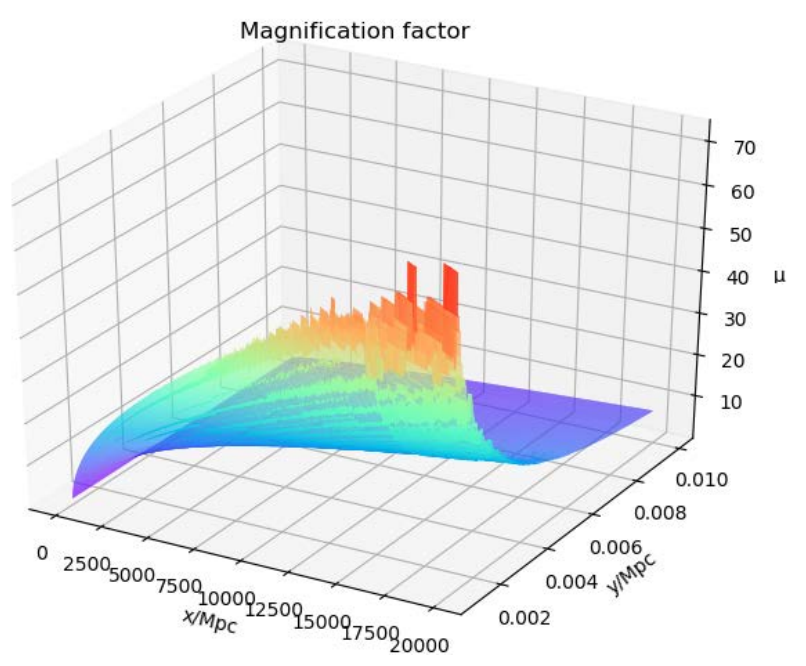

Fig.2

This figure shows the magnification factor calculated according to the formulas in section 2.3. It is obvious that gravitational lensing magnifies neutrino current significantly, especially when the observer is close to the optical axis, or x axis in this model. 


\section{Neutrino Oscillation in Propagation}

\subsection{Introduction to neutrino oscillation}

One of the most well-known peculiarities of neutrinos is their oscillation. There are three kinds of neutrino (electron neutrino, muon neutrino, and tau neutrino) in the standard model of particle physics. While propagating, one kind of neutrino might change into the other two kinds. This interesting phenomenon called neutrino oscillation accounts for the existence of the solar neutrino problem.

The mechanism of neutrino oscillation is too complicated to be showed here in detail. Alternatively, here is a picture showing the relation between the probability of oscillation, propagation distance, and the energy possessed by neutrinos. Since the nuclear reactions in astrophysical neutrino sources only generates electron neutrinos, the image which applies to neutrinos purely consists of electron neutrinos when start propagating is adopted:

Oscillation probabilities for an initial electron neutrino

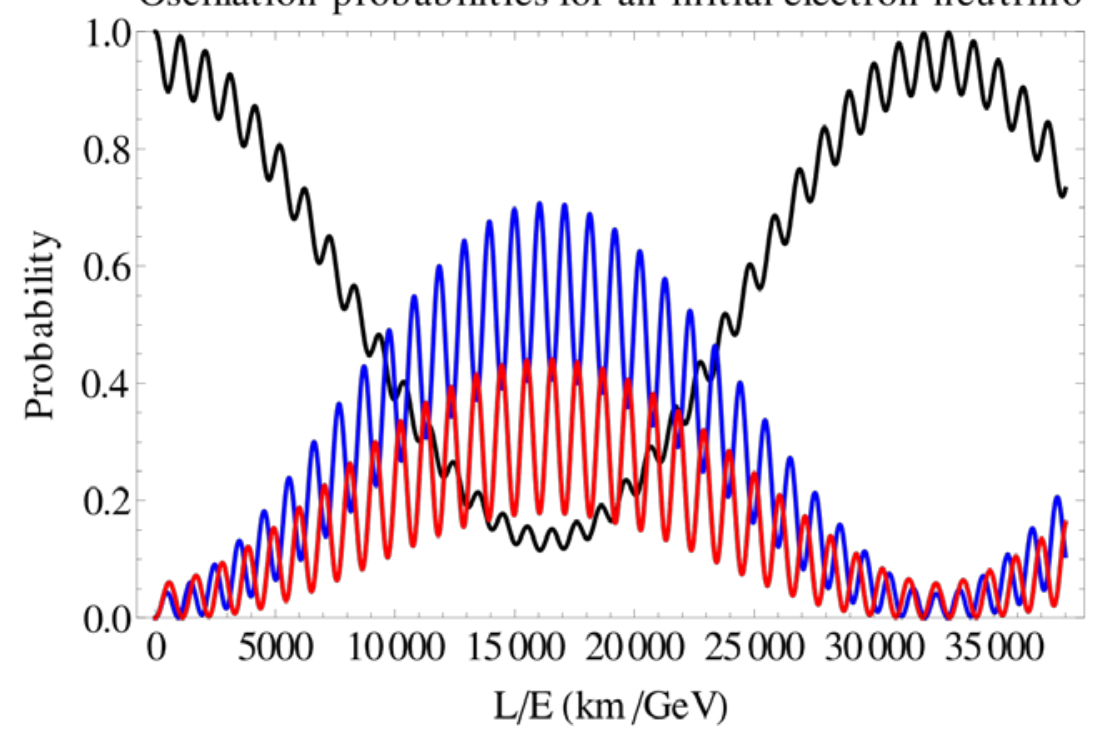

Fig.3

In the picture above, black, blue, and red lines stand for oscillation probability of electron, muon, and tau neutrinos, respectively. Source of the picture:

It can be seen in the picture that the variation of probability has a period of about $32000 \mathrm{~km} / \mathrm{GeV}$, i.e. a beam of electron neutrino flux carrying specific energy will turn into muon and tau neutrinos and then turn into electron neutrinos again after propagating for a specific distance.

\subsection{An example of neutrino oscillation in gravitational lensing}

In order to simplify the calculation of lengths of trajectories, a special condition that all the deflected neutrinos are brought to a point, as mentioned in section 2.2., should be considered. To show the effect of neutrino oscillation in gravitational lensing, we can take the famous supernova SN $1987 \mathrm{~A}$ as a typical source of neutrino flux. It is approximated that there are $10^{58}$ neutrinos carrying $10^{46} \mathrm{~J}$ of energy emitted. Assuming energy of all the neutrinos to be the same, each neutrino carries $6.25 \mathrm{MeV}$ of energy. In this case, the distance of one cycle of oscillation $l_{\text {cycle }}=32000 \mathrm{~km} / \mathrm{GeV} \times$ $6.25 \mathrm{MeV}=2 \times 10^{5} \mathrm{~m}$.

The distance between SN 1987A and Earth is 168000 ly, or 51.4 kpc. However, it is unlikely to find an enough massive object within this distance. In order to be consistent with the real condition where gravitational lensing is more possible to take place, consider the situation where the source is $1 \times 10^{5} \mathrm{Mpc}$ away from the observer.

Assume that there is an ideal mass sheet, located at the midpoint between the source and the observer, acting as a gravitational lens which can converge all the deflected neutrinos to Earth. It can be calculated that its surface-mass density 


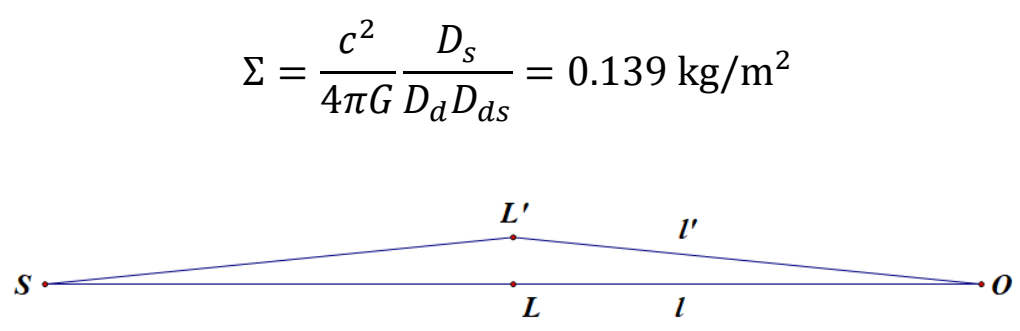

Fig.4

In this diagram, it is shown that neutrinos propagating through different paths travels differently long. $\mathrm{S}$ is the source, $\mathrm{L}$ is the location of the lens mass, and $\mathrm{O}$ is the observer. Two beams of neutrino flux set off at the source. One of them propagates directly to the observer. The other one set off by a separation of 1 arcsec (not drawn to scale) from optical axis SO, be deflected at point L' (as mentioned in section 2.1., the curved part of the path can be neglected), and then propagate to the observer.

For the beam of neutrino flux which propagates directly towards Earth, the length of its trajectory

$$
l=1 \times 10^{5} \mathrm{Mpc}
$$

For the other beam of neutrino flux which has an initial separation from the optical axis, the length of its trajectory

$$
l^{\prime}=\frac{1 \times 10^{5} \mathrm{Mpc}}{\cos 1^{\prime \prime}}
$$

The difference between these two lengths

$$
\Delta l=l^{\prime}-l=1 \times 10^{5} \mathrm{Mpc} \times\left(\frac{1}{\cos 1^{\prime \prime}}-1\right) \approx 3.626 \times 10^{16} \mathrm{~m} \approx 1.813 \times 10^{11} \times l_{\text {cycle }}
$$

It can be inferred that if we have a highly advanced detector which can precisely detect neutrino fluxes coming from different directions, we will find the composition of them to vary accordingly. This is just a simplified calculation via an idealized model, but this underlying phenomenon may actually facilitate our observation of the universe and the study of properties of neutrinos.

\section{Conclusion}

Gravitational lens, which is a well-known phenomenon predicted by the general theory of relativity, has been studied intensively and applied widely in astrophysics. However, the probability of applying gravitational lensing to the study of neutrino is seldom discussed. It can be seen in this paper that applying gravitational lensing to neutrino may yield many interesting results. The effect of neutrino oscillation may be showed in a special way in gravitational lensing. Due to the extreme inactivity of neutrinos, the detection of them is incredibly difficult. Since gravitational lens converges neutrino flux, magnifying it by a factor of ten or hundred, it may also facilitate neutrino detection. With advances of neutrino observing technique being made, the prospect of applying gravitational lensing to the study of neutrino is growing.

\section{References}

[1] Pereira J G, Zhang C M. LETTER: Some Remarks on the Neutrino Oscillation Phase in a Gravitational Field [J]. General Relativity \& Gravitation, 2000, 32(8):1633-1637.

[2] Gago A M, Nunokawa H, Funchal R Z. The Solar Neutrino Problem and Gravitationally Induced Long-wavelength Neutrino Oscillation [J]. Physical Review Letters, 2000, 84(18):4035-4038.

[3] Marek A, Janka H T, Mueller E. Equation-of-State Dependent Features in Shock-Oscillation 
Modulated Neutrino and Gravitational-Wave Signals from Supernovae [J]. Astronomy \& Astrophysics, 2009, 496(2):475-494.

[4] Ahluwalia D V, Burgard C. About the Interpretation of Gravitationally Induced Neutrino Oscillation Phases [J]. Science in China, 1996, 44(2):241-248.

[5] Luo X L. Neutrino Lensing [J]. Chinese Physics Letters, 2009, 26(10):224-227. 